\title{
Kinetics and Mechanism of the Hydrolysis and Rearrangement Processes within the Assembly-Disassembly-Organization- Reassembly Synthesis of Zeolites
}

\author{
Susan E. Henkelis, ${ }^{\dagger}$ Michal Mazur, ${ }^{\ddagger}$ Cameron M. Rice, ${ }^{\dagger}$ Paul S. Wheatley, ${ }^{\dagger}$ Sharon E. Ashbrook, ${ }^{\dagger}$ \\ and Russell E. Morris $*, \dagger, \ddagger$ \\ ${ }^{\dagger}$ School of Chemistry and EaStCHEM, University of St. Andrews, North Haugh, St. Andrews, Fife KY16 9ST, United Kingdom \\ ${ }^{\ddagger}$ Department of Physical and Macromolecular Chemistry Faculty of Sciences, Charles University, Hlavova 8, 12843 Prague 2, Czech \\ Republic
}

Supporting Information

ABSTRACT: The hydrolysis (disassembly, D) and rearrangement (organization, O) steps of the assembly-disassembly-organization-reassembly (ADOR) process for the synthesis of zeolites have been studied. Germanium-rich UTL was subjected to hydrolysis conditions in water to understand the effects of temperature (100, 92, 85, 81, 77, and $70{ }^{\circ} \mathrm{C}$ ). Samples were taken periodically over an $8-37 \mathrm{~h}$ period, and each sample was analyzed by powder X-ray diffraction. The results show that the hydrolysis step is solely dependent on the presence of liquid water, whereas the rearrangement is dependent on the temperature of the system. The kinetics have been investigated using the Avrami-Erofeev model. With increasing temperature, an increase in the rate of reaction for the rearrangement step was observed, and the Arrhenius equation was used to ascertain an apparent activation energy for the rearrangement from the kinetic product of the disassembly (IPC-1P) to the thermodynamic product of the rearrangement (IPC-2P). From this information, a mechanism for this transformation can be postulated.

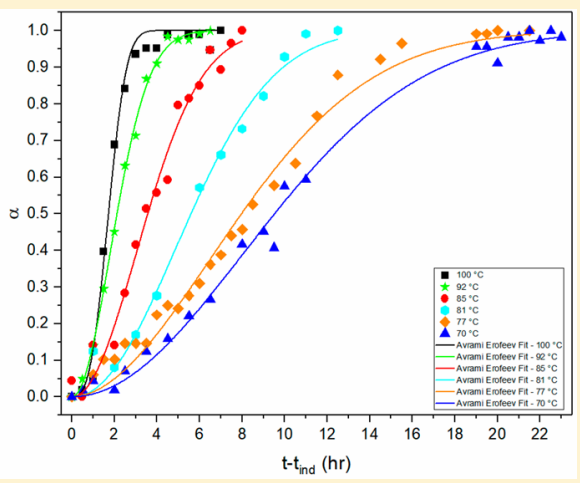

\section{INTRODUCTION}

A newly developed method for the synthesis of new zeolites coined the assembly-disassembly-organization-reassembly (ADOR) process has become well established in producing new zeolites that would not have been feasible through traditional routes (Scheme 1). ${ }^{1-7}$

Parent zeolites prepared for the ADOR process must have suitable chemical and physical properties in order to produce new daughter zeolites. Such properties include the presence of double four rings $(\mathrm{d} 4 \mathrm{r})$ with germanium preferentially located within them. ${ }^{8,9}$ Zeolites that have been successfully used in the ADOR process include UTL, ${ }^{2,6,7}$ ITH, $^{4}$ ITR, $^{4}$ IWR, $^{4}$ IWW, UOV, ${ }^{10,11}$ and recently discovered SAZ-1 ${ }^{12}$ (Figure 1). All zeolites that contain Ge-rich $\mathrm{d} 4 \mathrm{r}$ units, which we tested to date, can be successfully disassembled. However, not all have been successfully reassembled as yet. The layered intermediates that are formed on the disassembly of zeolites such as UTL seem to be relatively easy to manipulate to form new structures. However, these $\mathrm{d} 4 \mathrm{r}$-containing zeolites that disassemble into clusters (and not layers), such as polymorph $\mathrm{C}$ of zeolite beta, are much more difficult to organize and reassemble successfully.

The ADOR process has four main steps (Scheme 2). First, assembly (A) is the formation of the predetermined parent zeolite containing the required structural features. Research so far has centered on zeolites that consist of dense silicate layers connected by germanium-rich cubic $\mathrm{d} 4 \mathrm{r}$ building units.

The second step, disassembly (D), involves the removal of Ge-rich $\mathrm{d} 4 \mathrm{r}$ by aqueous or acid hydrolysis to produce a layered intermediate species (IPC-1P). The weakness in $\mathrm{Ge}-\mathrm{O}-\mathrm{Ge}$ and $\mathrm{Ge}-\mathrm{O}-\mathrm{Si}$ bonds allows for the facile hydrolysis of $\mathrm{Ge}$ and the collapse or "unzipping" of the $3 \mathrm{D}$ layered framework to form dense silicate-rich layers (Scheme 3). ${ }^{13,14}$

The layered species, IPC-1P, can then undergo several different fates depending on the choice of reaction conditions. First, it can be directly reassembled (R) to form a fully connected zeolite called IPC-4 (Scheme 4). To get a highly crystalline zeolite from the reassembly step, the IPC-1P has to be well organized, either through the intercalcation of a structure-directing-agent (SDA) or by leaving the IPC-1P for the right amount of time so that it self-organizes. Alternatively, it is possible to intercalate extra silicon between the layers to form a new zeolite precursor called IPC-2P. This zeolite can also form if IPC-1P is left under certain conditions. ${ }^{1,7,15,16}$ It is this process that is the major target of this particular article.

The ADOR process has previously been investigated using a range of different techniques, including solid-state NMR

Received: January 18, 2019

Published: February 20, 2019 
Scheme 1. Schematic of the First Confirmed Case of Utilizing the ADOR Process to Afford New Zeolites ${ }^{a}$

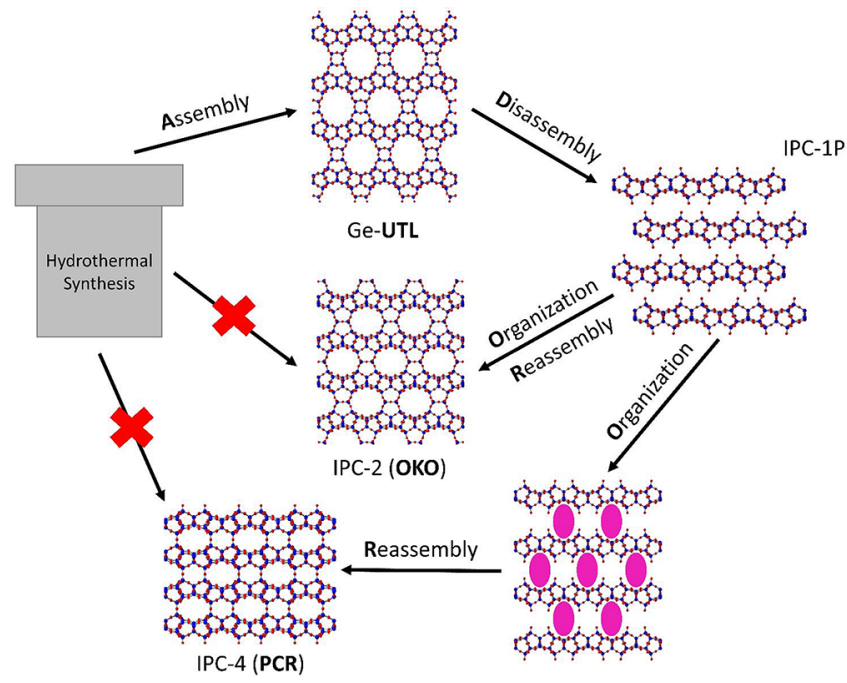

${ }^{a}$ Parent Ge-UTL is disassembled through hydrolysis into IPC-1P and then suitably rearranged into IPC-4 (PCR) or IPC-2 (OKO). IPC-4 is made up of direct oxygen linkages. IPC-2 consists of IPC-1P silicate layers connected by s4r. At the time of primary publication, these frameworks could not be made through traditional hydrothermal synthesis.

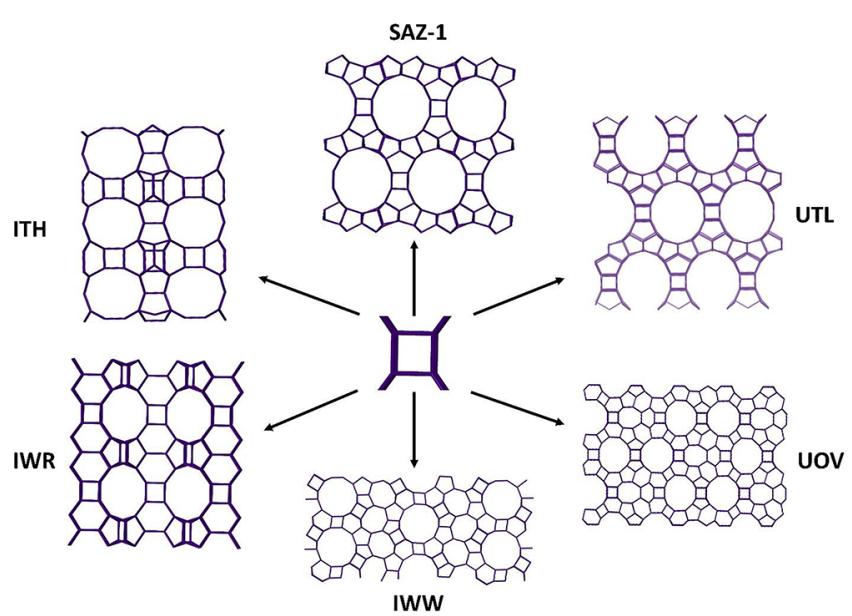

Figure 1. Double four ring $(\mathrm{d} 4 \mathrm{r})$ containing germanosilicates used successfully in the ADOR process.
Scheme 3. Hydrolysis of Ge-UTL over the Course of $1 \mathrm{~h}^{a}$

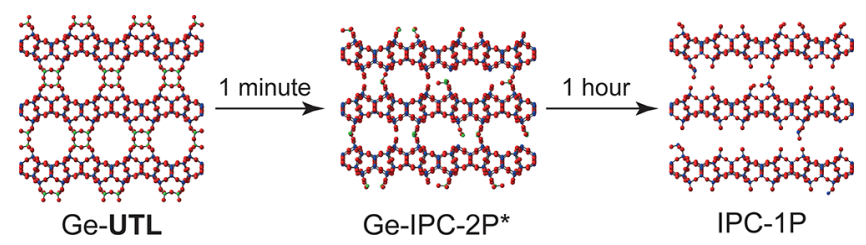

${ }^{a}$ After $1 \mathrm{~min}$, the hydrolysis is $60 \%$ complete with ca. $60 \%$ of the $\mathrm{d} 4 \mathrm{r}$ selectively hydrolyzed. After $1 \mathrm{~h}$, the hydrolysis has come to completion to form IPC-1P layers.

Scheme 4. Organization Step by Intercalation with a Structure-Directing Agent (Top) or a Hydrolysis/ Deintercalation and Self-Rearrangement in Acid (Bottom)

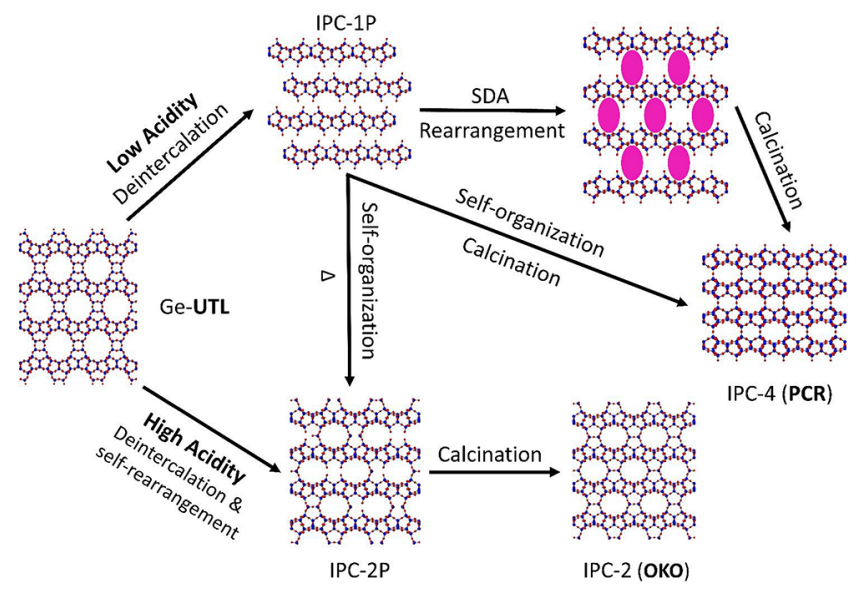

spectroscopy, ${ }^{17,18}$ in situ and ex situ pair distribution function (PDF) analysis, ${ }^{19,20}$ and both in situ and ex situ powder X-ray diffraction (PXRD). ${ }^{12,20,21}$

For the first time, we present a kinetic analysis of the two most prominent steps ( $\mathrm{D}$ and $\mathrm{O}$ ) in the ADOR process, found by sampling the reaction and using powder X-ray diffraction to follow the evolution of the lattice spacing with time. Each data set was fit with the Avrami-Erofeev model, and the activation energy of the rearrangement (organization) step was found to be $70.1 \mathrm{~kJ} \mathrm{~mol}^{-1}$.

\section{MATERIALS AND METHODS}

Synthesis of Parent Ge-UTL with Molar Composition $0.8 \mathrm{GeO}_{2}: 0.4 \mathrm{ROH}: 30 \mathrm{H}_{2} \mathrm{O}$. Germanium dioxide $(7.75 \mathrm{~g})$ was dissolved in an aqueous solution of $(6 R, 10 S)$-6,20-dimethyl-5azoniaspiro[4,5]decane hydroxide $\left(12.7 \mathrm{~g}, \mathrm{H}_{2} \mathrm{O} 100 \mathrm{~mL}, 0.625 \mathrm{M}\right)$.

Scheme 2. ADOR Mechanism of IPC-2 Zeolite Synthesis ${ }^{a}$

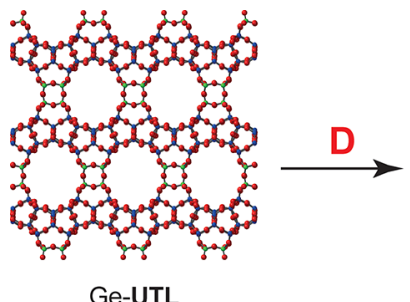

Ge-UTL

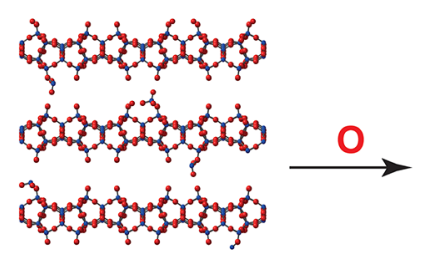

IPC-1P

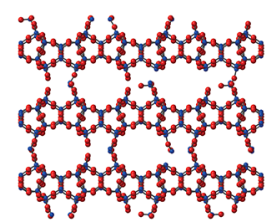

IPC-2P

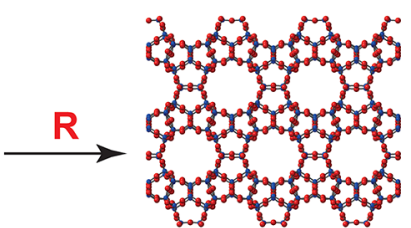

IPC-2

(OKO)

\footnotetext{
${ }^{a}$ Assembly: predetermined parent zeolite Ge-UTL is produced. Disassembly: germanium is selectively hydrolyzed, leading to the breakdown of the $\mathrm{d} 4 \mathrm{r}$ to form layered material IPC-1P. Organization: the IPC-1P layers are suitably reorientated through a self-organization process to form IPC-2P. Reassembly: new silicate bonds are formed between the layers to afford IPC-2 upon calcination. Si, blue; Ge, green; and O, red.
} 
Fumed silicon dioxide $(8.90 \mathrm{~g})$ was added portionwise to the mixture over 30 min until a homogeneous solution was formed. The gel was transferred to a Teflon-lined steel autoclave and held at $175{ }^{\circ} \mathrm{C}$ for 7 days. The zeolite product was collected by filtration, washed with water $(200 \mathrm{~mL})$, and dried at $80{ }^{\circ} \mathrm{C}$ for $12 \mathrm{~h}$. To remove the SDA, the as-synthesized zeolite was calcined in a stream of air at $575^{\circ} \mathrm{C}$ for $7 \mathrm{~h}$ with a temperature ramp of $1{ }^{\circ} \mathrm{C} \mathrm{min}^{-1}$.

Hydrolysis Procedure. All Ge-UTL used in each reaction was afforded from one synthesis batch.

Ge-UTL $(600 \mathrm{mg})$ was added to water $(120 \mathrm{~mL})$ with stirring at the desired temperature. The reaction mixture was stirred at the desired temperature with aliquots taken every $1 \mathrm{~min}$ for $5 \mathrm{~min}$, every 5 min for up to $1 \mathrm{~h}$, and then every 30 min until the rearrangement had gone to completion $(8-37 \mathrm{~h})$. The collected samples were filtered, dried on the filter for $1 \mathrm{~min}$, and then dried at $80^{\circ} \mathrm{C}$ for $5 \mathrm{~min}$.

Characterization Techniques. Laboratory powder X-ray diffraction data were collected on both a Panalytical Emperean diffractometer monochromated with a curved $\mathrm{Ge}(111)$ crystal in reflectance mode and a STOE STADIP operated in capillary DebyeScherrer mode. Both diffractometers operated with $\mathrm{Cu} \mathrm{K} \alpha 1$ radiation.

Kinetic Analysis. The temperature dependence of the hydrolysis (D) and rearrangement $(\mathrm{O})$ processes in water was investigated over a temperature range of $70-100{ }^{\circ} \mathrm{C}$ using the Avrami-Erofeev model (eq 1) and its linear equivalent, the Sharp Hancock equation (eq 2).

$$
\begin{aligned}
& {[-\ln (1-\alpha)]^{1 / n}=k\left(t-t_{\text {ind }}\right)} \\
& \ln [-\ln (1-\alpha)]=n \ln (k)+n \ln (\mathrm{t})
\end{aligned}
$$

The effect of temperature on the system was investigated on 100 , $92,85,81,77$, and $70^{\circ} \mathrm{C}$. Samples were taken periodically over an $8-$ $37 \mathrm{~h}$ period, and analyzed by powder X-ray diffraction to determine the level of reaction completion and the daughter zeolite being produced (Figure 2). Each sample at the designated time set was analyzed by PXRD, and the location of the $d_{200}$ peak was recorded. This peak represents the interlayer distance between the dense silicate-rich layers and as such provides valuable information on the status of the reaction, allowing one to elucidate the level of completion at that time and the rate of reaction for each data set.

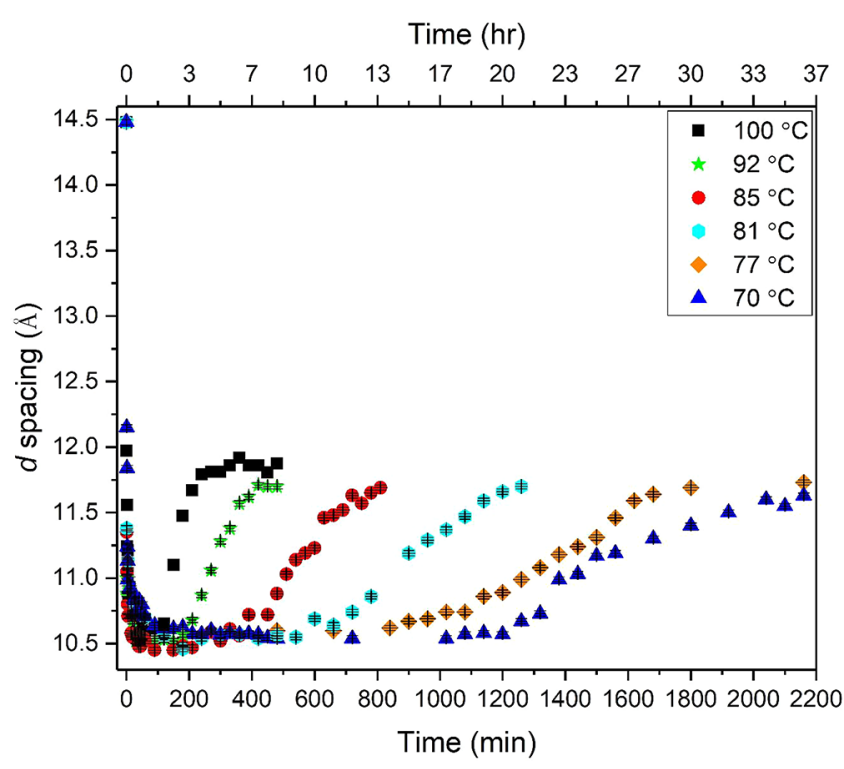

Figure 2. Change in $d_{200}$ interlayer spacing for the hydrolysis and rearrangement steps for the reaction of Ge-UTL in water with increasing temperature: $100{ }^{\circ} \mathrm{C}$, black squares; $92^{\circ} \mathrm{C}$, green stars; 85 ${ }^{\circ} \mathrm{C}$, red circles; $81{ }^{\circ} \mathrm{C}$, teal pentagons; $77^{\circ} \mathrm{C}$, orange diamonds; and $70{ }^{\circ} \mathrm{C}$, blue triangles. All data points were fit with an error of $\pm 0.2 \AA$. See reference 31 for full protocol.
The Avrami-Erofeev model is well established for modeling solidstate kinetics as it can specifically describe the kinetics for crystallization and the method and direction of growth of the nucleates. It is well known to be used to monitor phase transitions and understand the mechanism of intercalation/rearrangement processes. $^{22-28}$

For each reaction carried out, the Avrami-Erofeev model was fitted to experimental data to calculate the reaction exponent, $n$, and the rate constant, $k$. The extent of reaction, $\alpha$, was measured using the change in the interlayer $d$ spacing normalized to between 0 and 1 .

\section{RESULTS AND DISCUSSION}

Hydrolysis of Ge-UTL. The hydrolysis of Ge-UTL in water can be mapped through PXRD, and a change in $d$ spacing from UTL (14.48 $\AA$ ) to disordered layered material IPC-1P (10.54 $\AA$ ) can be clearly seen. The extent of reaction vs time was plotted for the reactions run at $100,92,85,81,77$, and $70{ }^{\circ} \mathrm{C}$, and each plot was fitted with the Avrami-Erofeev model (Figure 3). The ratio of zeolite/water is an important factor to

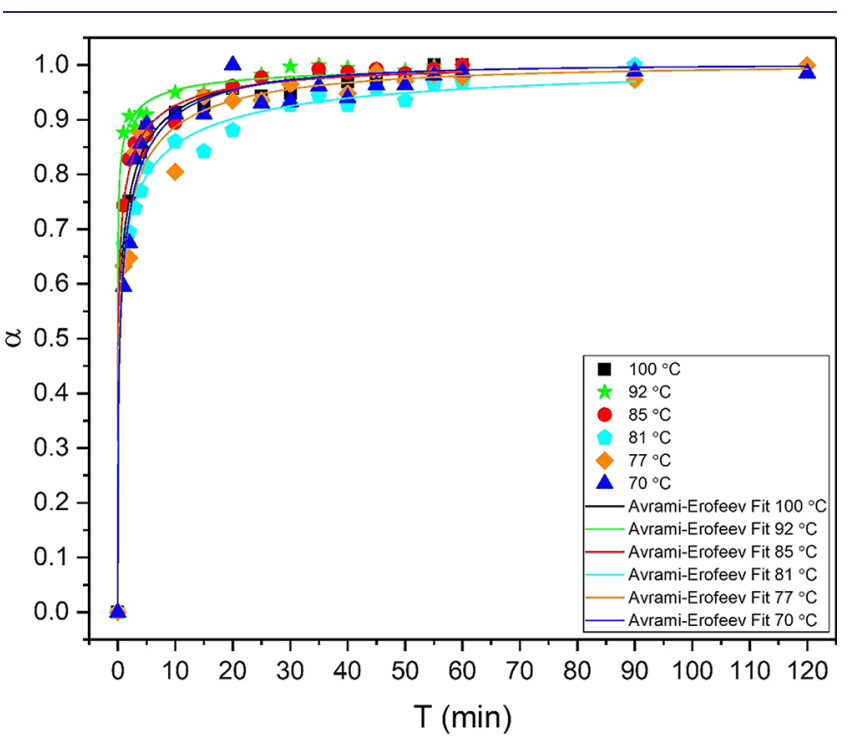

Figure 3. Extent of reaction, $\alpha$, plotted against time for the hydrolysis (D) step: $100{ }^{\circ} \mathrm{C}$, black squares; $92^{\circ} \mathrm{C}$, green stars; $85^{\circ} \mathrm{C}$, red circles; $81^{\circ} \mathrm{C}$, teal pentagons; $77^{\circ} \mathrm{C}$, orange diamonds; $70^{\circ} \mathrm{C}$, blue triangles. Each plot was fitted with the Avrami-Erofeev model.

be considered because significantly reducing the amount of water effectively reduces the rate of hydrolysis (D), and under low-volume conditions $(1 \mathrm{~g} / 8 \mathrm{~mL})$, the material never fully hydrolyzes to IPC-1P. In this work, a ratio of $1 \mathrm{~g} / 200 \mathrm{~mL}$ for zeolite/water was used to ensure full hydrolysis.

From both the initial PXRD data and the subsequent Avrami plots, it is clear to see that the hydrolysis step is not dependent on the temperature of the reaction system. The Avrami exponent $n\left(n_{\mathrm{AE}}\right)$ was found to be $<1$ for each temperature (Table 1 in the SI). An Avrami exponent that is this low can normally be attributed to diffusion-controlled 1D growth. In this case, because the rate of reaction is so fast and we see a $60 \%$ collapse of $\mathrm{d} 4 \mathrm{r}$ within $1 \mathrm{~min}$, the results from the fit are unsuitable for further analysis. The minor changes in reaction time can be attributed to human error, such as changes in mixing and time of addition of the parent zeolite.

Although the rate constant $k\left(k_{\mathrm{AE}}\right)$ is unreliable, because of the rapid rate of hydrolysis/deintercalation (as seen by a change in $d$ spacing), we can assume that the mechanism proceeds without having to overcome a high activation barrier, 
again suggesting that temperature is not a requirement for the hydrolysis and solely depends on the availability of liquid water.

As $\mathrm{d} 4 \mathrm{r}$ collapse, the material moves through a new species we coin Ge-IPC-2P* (formed after $1 \mathrm{~min}$ ). This is effectively a single four ring ( $\mathrm{s} 4 \mathrm{r}$ ) containing IPC-2P structure but with germanium still residing between the layers, creating local disorder ( $\mathrm{Si} / \mathrm{Ge} 10.7)$. As such, this material has a broad $d_{200}$ peak in the XRD patterns.

Occasionally described as a more reliable solid-state model for finding $n$, the Sharp Hancock method ${ }^{29}$ (SI Figure 1) was applied by taking natural logarithms of the Avrami-Erofeev equation and the values of $n\left(n_{\mathrm{SH}}\right)$ and $k\left(k_{\mathrm{SH}}\right)$ compared for each model (SI Table 1). Again, because of the reaction rate, the results obtained are unsuitable and further analysis using in situ techniques is needed to monitor the hydrolysis mechanism on a second time scale.

Rearrangement to IPC-2P. Once the hydrolysis from $\mathrm{Ge}$ UTL to IPC-1P was complete, various induction times can be seen before the full rearrangement to IPC-2P through an the IPC-6P intermediate. IPC-6P is a $44 \mathrm{r}$ and direct oxygen linkage containing daughter zeolite. As such, it contains layers of IPC$2 \mathrm{P}$ and layers of IPC-1P (Figure 4). Because of this, the $d_{200}$ peak in the powder pattern becomes broader

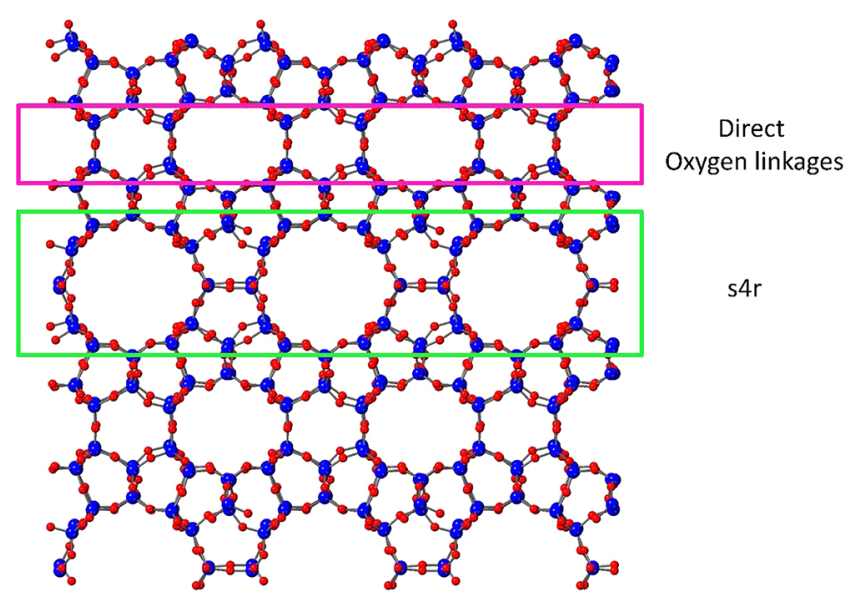

Figure 4. IPC-6 framework with alternating layers of s4r and direct oxygen bridges. s4r, green box; direct oxygen bridges, pink box.

A clear temperature dependence on the system can be seen with reaction times increasing from 2 to $22 \mathrm{~h}$ as the temperature decreases from 100 to $70{ }^{\circ} \mathrm{C}$. As the temperature increases, the time taken to induct decreases dramatically, with $70{ }^{\circ} \mathrm{C}$ inducting for $20 \mathrm{~h}$ and $100{ }^{\circ} \mathrm{C}$ inducting for only $1 \mathrm{~h}$.

The Avrami-Erofeev model was fit to the extent of reaction data, where $t-t_{\text {ind }}$ was taken to be the point at which the induction came to an end and the intercalation began (Figure 5).

The Avrami exponent, $n$, was found to be 3 for $100{ }^{\circ} \mathrm{C}$ and 2 for all lower temperatures. Because the atomic nuclei are preformed in all cases, the growth is restricted to three dimensions $(n=3)$ and two dimensions $(n=2)$. As such, the rearrangement of silicates into the layers is occurring along the $x, y$, and $z$ axes when $n=3$, ultimately forming a $3 \mathrm{D}$ connected framework. However, when $n=2$, rearrangement occurred only along the $x / y, x / z$, or $y / z$ axes at any one time, which will slow the rate of forming the fully connected 3D true zeolite. The rate constant, $k$, decreases from $0.510 \mathrm{~min}^{-1}$ at $100{ }^{\circ} \mathrm{C}$ to

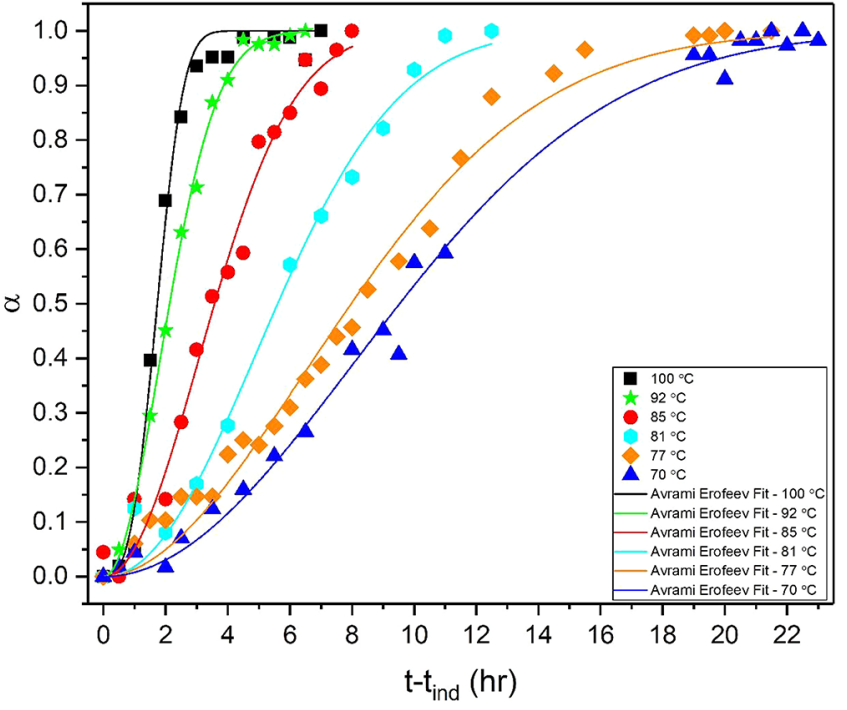

Figure 5. Extent of reaction, $\alpha$, plotted against time for the rearrangement (O) step: $100{ }^{\circ} \mathrm{C}$, black squares; $92{ }^{\circ} \mathrm{C}$, green stars; $85{ }^{\circ} \mathrm{C}$, red circles; $81{ }^{\circ} \mathrm{C}$, teal pentagons; $77^{\circ} \mathrm{C}$, orange diamonds; and $70^{\circ} \mathrm{C}$, blue triangles. Each plot was fitted to the Avrami-Erofeev model.

$0.391,0.233,0.150,0.103$, and $0.087 \mathrm{~min}^{-1}$ at $92,85.81,77$, and $70{ }^{\circ} \mathrm{C}$, respectively. The data was once again compared against the Sharp Hancock model, and the values for $n$ and $k$ were compared (SI Figure 2 and Table 2).

The activation energy, $E_{\mathrm{a}}$, was calculated to be $70.1 \mathrm{~kJ} \mathrm{~mol}^{-1}$ (Figure 6). This was extrapolated from a plot of $\ln (k)$ against

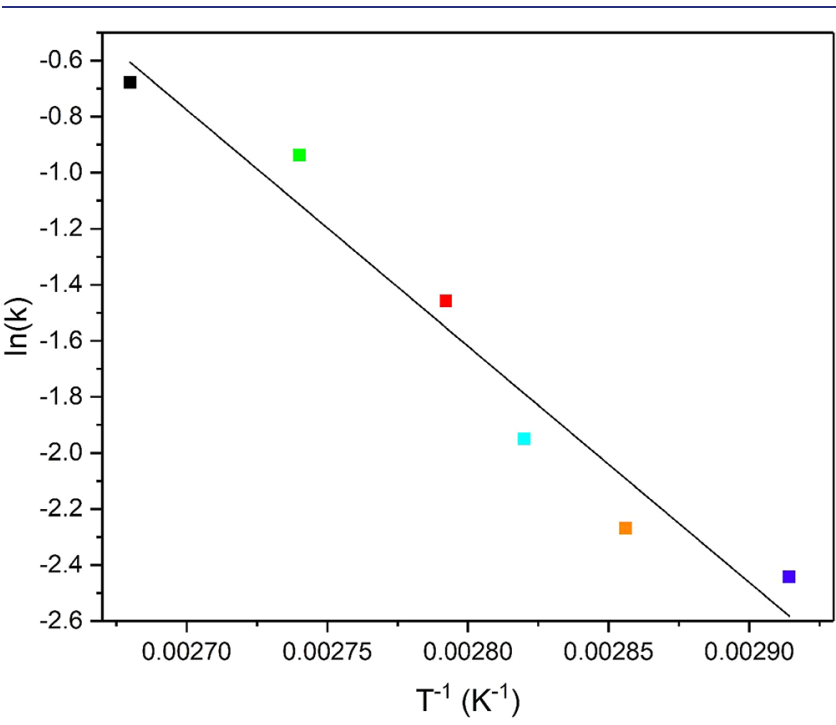

Figure 6. Arrhenius plot of $\ln (k)$ vs $T^{-1}$ to attain an activation energy of $70.1 \mathrm{~kJ} \mathrm{~mol}^{-1}$ for the rearrangement from IPC-1P to IPC-2P.

$T^{-1}$ based on the Arrhenius equation (eq 3). This activation energy is relatively high in comparison to the intercalation mechanisms of other layered materials. For example, the activation energy required for chlorophenoxyacetates to intercalate (rearrange) into double-layered hydroxides is 43 , 53.6 , and $61.7 \mathrm{~kJ} \mathrm{~mol}^{-1}$. The increase in activation energy is linearly dependent on the size of the chlorophenoxyacetate introduced. This suggests that the energy needed for silicates to rearrange into the layers is high and may be due to the close 
proximity of the IPC-1P hydrogen-bonded layers. The values of $k$ were taken from the Avrami fittings.

$$
k=A \mathrm{e}^{-E_{\mathrm{a}} / R T}
$$

From Where Does the Silicon Rearrange? Understanding from where the silicon rearranges is imperative to fully understanding the mechanism. An experiment was devised to quantify whether the silicon rearranged from silicates present in the solution after hydrolysis or from the silicon in the silica-rich layers.

Using the $100{ }^{\circ} \mathrm{C}$ reaction in water as a standard, once hydrolysis was complete at $1 \mathrm{~h} 30 \mathrm{~min}$, the suspension was filtered to remove the silicate-rich solution and replaced with fresh water at a certain temperature (known herein as $100{ }^{\circ} \mathrm{C}$ with fresh water). The removal of the silicate solution leads to a change in the IPC-1P material, which is likely to be a subzeolite $^{30}$ in the first instance, with a small interlayer distance. This material is similar to a solid termed IPC-1, which was the first microporous zeolite to be produced through ADOR. IPC-1 is a very disordered structure that is produced from the disassembly of B-UTL after calcination. ${ }^{6}$ This material can be visualized by the interlocking of one's fingers, where the palms of the hands are the silica-rich layers and fingers are the silanol groups on the surface of the layers. After reacting for another $5 \mathrm{~h}$, the layers have rearranged to IPC-2P (Figure 7).

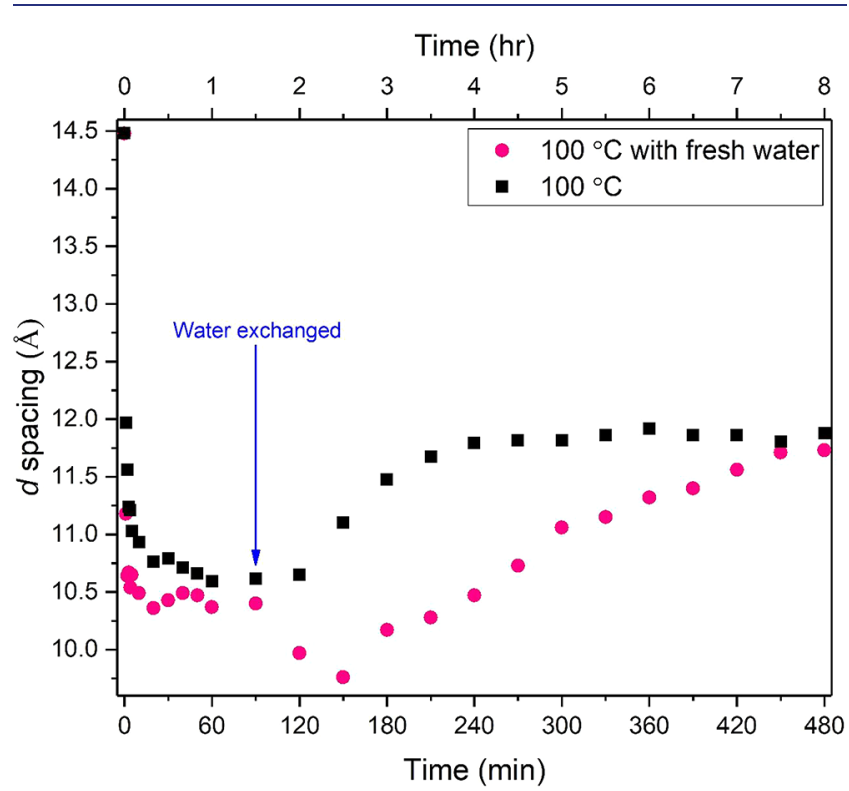

Figure 7. Change in $d_{200}$ interlayer spacing for the hydrolysis and rearrangement steps: $100{ }^{\circ} \mathrm{C}$, black squares; $100{ }^{\circ} \mathrm{C}$ with fresh water replaced at $90 \mathrm{~min}$, pink circles.

As all sources of silicon in solution have been removed, we can say that the silicon must be rearranging from the siliconrich layers, thus potentially causing defect sites (Scheme 5).

The change in reaction time can be quantified. When the silicon rearranges from the layers, the time taken to rearrange from IPC-1P increases from 2 to $6 \mathrm{~h} 30 \mathrm{~min}$, thus affirming that the rearrangement from silicates present in solution due to the breakdown of the $\mathrm{d} 4 \mathrm{r}$ is the preferred method. The kinetics were once again analyzed by the Avrami-Erofeev model (Figure 8), and the Avrami exponent $n$, for $100{ }^{\circ} \mathrm{C}$ fresh water was found to be 1 , thereby controlling the growth in only one
Scheme 5. Change in Structure with a Change in Solution ${ }^{a}$

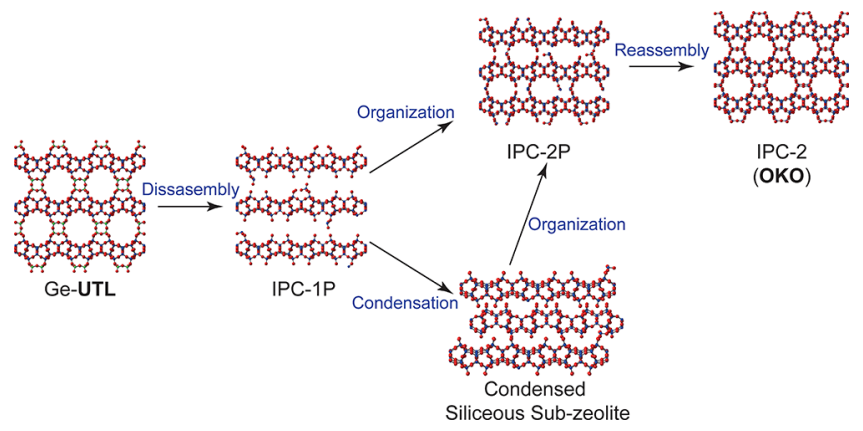

${ }^{a}$ Disassembly: Ge-UTL is first hydrolyzed to IPC-1P. Organization: The layers then self-rearrange to IPC-2P. Condensation: The layers condense further to form a subzeolite of IPC-1P, termed IPC-1, and then self-rearrange to IPC-2P. Reassembly: Formation of silicate bonds between IPC-2P to form IPC-2.

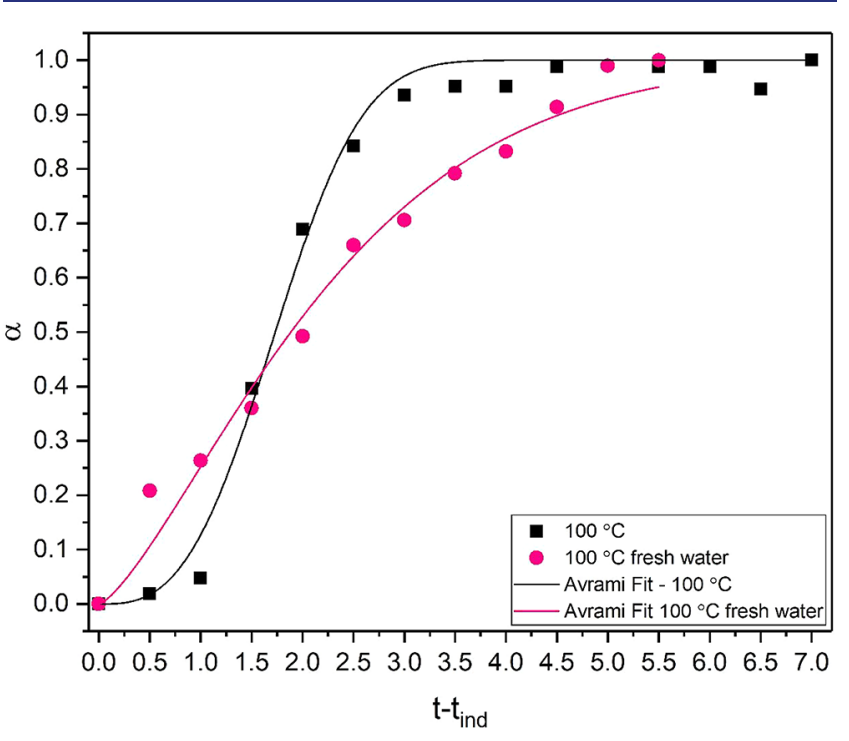

Figure 8. Extent of reaction, $\alpha$, plotted against time for the rearrangement (O) step: $100{ }^{\circ} \mathrm{C}$, black squares; $100{ }^{\circ} \mathrm{C}$ fresh water; pink circles. Both plots were fitted with the Avrami-Erofeev model.

dimension. The rate constant, $k$, was found to be similar to that of the $92{ }^{\circ} \mathrm{C}$ reaction, with a rate constant of $0.405 \mathrm{~min}^{-1}$.

As rearrangement to IPC-2P is favored even when there are no silica species present, we can conclude that IPC-1P is the kinetic product and IPC-2P is the thermodynamic product (Scheme 6).

\section{CONCLUSIONS}

Germanium-rich UTL was subjected to hydrolysis conditions in water as the medium to understand the effects of temperature $\left(100,92,85,81,77\right.$, and $\left.70{ }^{\circ} \mathrm{C}\right)$. Solid-state kinetic models, Avrami-Erofeev, and Sharp Hancock were employed and it was found that the kinetics of hydrolysis (D) is not dependent on the temperature of the reaction system but is solely dependent on the presence of liquid water. The rearrangement process, however, is directly dependent on temperature, and with increasing temperature, an increase in the rate was observed. Through use of the Avrami-Erofeev model, $n$ was found to be 3 for $100{ }^{\circ} \mathrm{C}, 2$ for all other temperatures, and 1 when the silicate-rich solution is replaced 
Scheme 6. Each Step in the ADOR Process for Ge-UTL when Hydrolysis Is Carried out at $100{ }^{\circ} \mathrm{C}$ in Water, with All Intermediate Materials Shown ${ }^{a}$

Disassembly

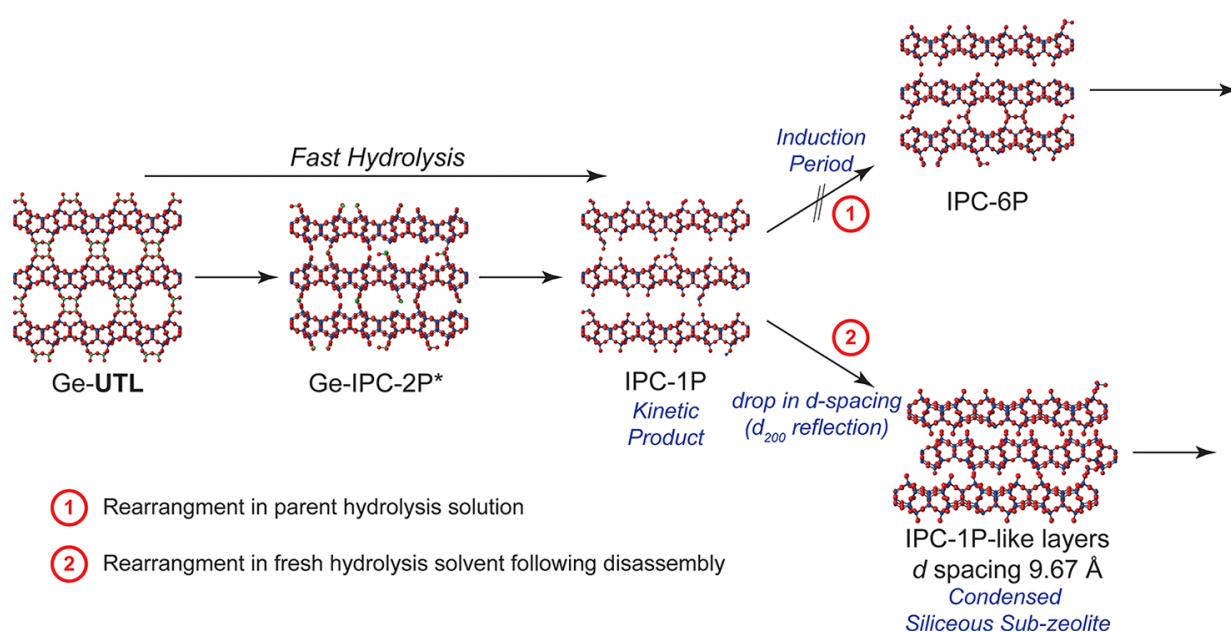

Organization

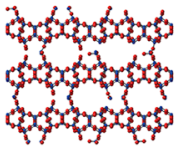

IPC-2P

$$
\begin{aligned}
& \text { Thermodynamic } \\
& \text { Product }
\end{aligned}
$$
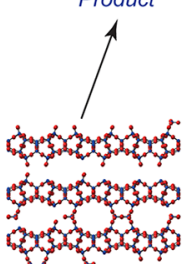

IPC-6P

Reassembly

${ }^{a}$ Pathway 1 shows the rearrangement when silicates are present in solution. Pathway 2 shows the rearrangement following replacement with fresh water at $1 \mathrm{~h} 30 \mathrm{~min}$.

with fresh water, thus confining the growth to one, two, and three dimensions when $n=1,2$, and 3, respectively. The activation energy of the rearrangement step was $70.1 \mathrm{~kJ} \mathrm{~mol}^{-1}$. When no silicates are present in solution, the silicon rearranges from the silica-rich layers and may cause defect sites throughout. As such, IPC-1P is the kinetic product and IPC$2 \mathrm{P}$ is the thermodynamic product.

The results we see from the kinetics study we describe here are supported by other experimental techniques, and this gives us great confidence that the protocol and analysis we provide give a good description of the process as a whole. For example, aside from the PXRD data shown here, we have monitored the ADOR process using NMR and TEM, as presented in ref 31 . Using ${ }^{29} \mathrm{Si} N M R$, the intensity of the $\mathrm{Q}^{4}$ and $\mathrm{Q}^{3}$ peaks was analyzed. The growth of the $\mathrm{Q}^{3}$ sites after $1 \mathrm{~h}$ suggested the formation of silanol groups on the surface of the layers after disassembly. After $1 \mathrm{~h}$, the $\mathrm{Q}^{3}$ peak then reduced as the layers rearranged themselves. TEM was consistent with the XRD, with a drop in $d$ spacing over $1 \mathrm{~h}$ before increasing again to form IPC-2P. In previous papers, we have discussed the importance of investigating the local structure of the materials produced through ADOR. We have shown that by using both in situ PDF and NMR the ADOR process can be monitored and that the synthesis procedure needs to be strictly monitored in order to produce consistent and comparable results. We have seen the fast hydrolysis and induction period in these studies, but because of the limitations of each technique, we have never been able to show the whole $\mathrm{D}$ and $\mathrm{O}$ processes in one analysis. By carefully developing this protocol and using the $d$ spacing as a marker for the different structures present, we have now shown that we can propose a mechanism that is supported by all the other studies.

\section{ASSOCIATED CONTENT}

\section{S Supporting Information}

The Supporting Information is available free of charge on the ACS Publications website at DOI: 10.1021/jacs.9b00643.
Sharp Hancock plots of the hydrolysis and rearrangement processes and comparison tables of Avrami vs Sharp Hancock data (PDF)

\section{AUTHOR INFORMATION}

\section{Corresponding Author}

*rem1@st-andrews.ac.uk

ORCID

Susan E. Henkelis: 0000-0003-4586-3881

Michal Mazur: 0000-0001-5044-5284

Sharon E. Ashbrook: 0000-0002-4538-6782

Notes

The authors declare no competing financial interest.

\section{ACKNOWLEDGMENTS}

The authors thank the EPSRC (grants EP/K025112/1, EP/ K005499/1, EP/K503162/1, and EP/N509759/1) for funding opportunities. R.E.M. and M.M. acknowledge OP VVV "Excellent Research Teams", project no. CZ.02.1.01/0.0/0.0/ 15_003/0000417-CUCAM. We also thank the ERC (advanced grant 787073 “ADOR").

\section{REFERENCES}

(1) Eliášová, P.; Opanasenko, M. V.; Wheatley, P. S.; Shamzhy, M. V.; Mazur, M.; Nachtigall, P.; Roth, W. J.; Morris, R. E.; Čejka, J. The ADOR Mechanism for the Synthesis of New Zeolites. Chem. Soc. Rev. 2015, 44 (20), 7177-7206.

(2) Mazur, M.; Wheatley, P. S.; Navarro, M.; Roth, W. J.; Položij, M.; Mayoral, A.; Eliášová, P.; Nachtigall, P.; Cejka, J.; Morris, R. E. Synthesis of "unfeasible" Zeolites. Nat. Chem. 2016, 8 (1), 58-62.

(3) Opanasenko, M. V.; Roth, W. J.; Čejka, J. Two-Dimensional Zeolites in Catalysis: Current Status and Perspectives. Catal. Sci. Technol. 2016, 6 (8), 2467-2484.

(4) Shamzhy, M.; Opanasenko, M.; Tian, Y.; Konysheva, K.; Shvets, O.; Morris, R. E.; C ejka, J. Germanosilicate Precursors of ADORable Zeolites Obtained by Disassembly of ITH, ITR, and IWR Zeolites. Chem. Mater. 2014, 26 (19), 5789-5798.

(5) Chlubná-Eliášová, P.; Tian, Y.; Pinar, A. B.; Kubů, M.; Čejka, J.; Morris, R. E. The Assembly-Disassembly-Organization-Reassembly 
Mechanism for 3D-2D-3D Transformation of Germanosilicate IWW Zeolite. Angew. Chem. 2014, 126 (27), 7168-7172.

(6) Roth, W. J.; Shvets, O. V.; Shamzhy, M.; Chlubna', P.; Kubu, M.; Nachtigall, P.; Čejka, J. Postsynthesis Transformation of ThreeDimensional Framework into a Lamellar Zeolite with Modifiable Architecture. J. Am. Chem. Soc. 2011, 133 (16), 6130-6133.

(7) Roth, W. J.; Nachtigall, P.; Morris, R. E.; Wheatley, P. S.; Seymour, V. R.; Ashbrook, S. E.; Chlubná, P.; Grajciar, L.; Položij, M.; Zukal, A.; Shvets, O.; Čejka, J. A Family of Zeolites with Controlled Pore Size Prepared Using a Top-down Method. Nat. Chem. 2013, 5 (7), 628-633.

(8) Corma, A.; Díaz-Cabañas, M. J.; Rey, F.; Nicolopoulus, S.; Boulahya, K. ITQ-15: The First Ultralarge Pore Zeolite with a BiDirectional Pore System Formed by Intersecting 14- and 12-Ring Channels, and Its Catalytic Implications. Chem. Commun. 2004, 0 (12), 1356-1357.

(9) Corma, A.; Fornés, V.; Martı'nez-Triguero, J.; Pergher, S. B. Delaminated Zeolites: Combining the Benefits of Zeolites and Mesoporous Materials for Catalytic Uses. J. Catal. 1999, 186 (1), $57-63$.

(10) Kasneryk, V.; Opanasenko, M.; Shamzhy, M.; Musilová, Z.; Avadhut, Y. S.; Hartmann, M.; Čejka, J. Consecutive Interlayer Disassembly-reassembly during Alumination of UOV Zeolites: Insight into the Mechanism. J. Mater. Chem. A 2017, 5 (43), $22576-22587$.

(11) Kasneryk, V.; Shamzhy, M.; Opanasenko, M.; Wheatley, P. S.; Morris, S. A.; Russell, S. E.; Mayoral, A.; Trachta, M.; Čejka, J.; Morris, R. E. Expansion of the ADOR Strategy for the Synthesis of Zeolites: The Synthesis of IPC-12 from Zeolite UOV. Angew. Chem., Int. Ed. 2017, 56 (15), 4324-4327.

(12) Firth, D. S.; Morris, S. A.; Wheatley, P. S.; Russell, S. E.; Slawin, A. M. Z.; Dawson, D. M.; Mayoral, A.; Opanasenko, M.; Položij, M.; Čjka, J.; Nachtigall, P.; Morris, R. E. Assembly-DisassemblyOrganization-Reassembly Synthesis of Zeolites Based on Cfi-Type Layers. Chem. Mater. 2017, 29 (13), 5605-5611.

(13) Kamakoti, P.; Barckholtz, T. A. Role of Germanium in the Formation of Double Four Rings in Zeolites. J. Phys. Chem. C 2007, 111 (9), 3575-3583.

(14) Wheatley, P. S.; Čejka, J.; Morris, R. E. Synthesis of Zeolites Using the ADOR (Assembly-Disassembly-Organization-Reassembly) Route. J. Visualized Exp. 2016, No. 110, e53463.

(15) Mazur, M.; Chlubná-Eliášová, P.; Roth, W. J.; Čejka, J. Intercalation Chemistry of Layered Zeolite Precursor IPC-1P. Catal. Today 2014, 227, 37-44.

(16) Shamzhy, M.; Mazur, M.; Opanasenko, M.; Roth, W. J.; Čejka, J. Swelling and Pillaring of the Layered Precursor IPC-1P: Tiny Details Determine Everything. Dalton Trans. 2014, 43 (27), 10548.

(17) Bignami, G. P. M.; Dawson, D. M.; Seymour, V. R.; Wheatley, P. S.; Morris, R. E.; Ashbrook, S. E. Synthesis, Isotopic Enrichment, and Solid-State NMR Characterization of Zeolites Derived from the Assembly, Disassembly, Organization, Reassembly Process. J. Am. Chem. Soc. 2017, 139 (14), 5140-5148.

(18) Morris, S. A.; Bignami, G. P. M.; Tian, Y.; Navarro, M.; Firth, D. S.; Čejka, J.; Wheatley, P. S.; Dawson, D. M.; Slawinski, W. A.; Wragg, D. S.; Morris, R. E.; Ashbrook, S. E. In Situ Solid-State NMR and XRD Studies of the ADOR Process and the Unusual Structure of Zeolite IPC-6. Nat. Chem. 2017, 9, 1012.

(19) Henkelis, S. E.; Morris, S. A.; Mazur, M.; Wheatley, P. S.; McHugh, L. N.; Morris, R. E. Monitoring the Assemblydisassembly-organisation-reassembly Process of Germanosilicate UTL through in Situ Pair Distribution Function Analysis. J. Mater. Chem. A 2018, 6 (35), 17011-17018.

(20) Morris, S. A.; Wheatley, P. S.; Položij, M.; Nachtigall, P.; Eliášová, P.; Cejka, J.; Lucas, T. C.; Hriljac, J. A.; Pinar, A. B.; Morris, R. E. Combined PDF and Rietveld Studies of ADORable Zeolites and the Disordered Intermediate IPC-1P. Dalt. Trans. 2016, 45 (36), 14124-14130.

(21) Wheatley, P. S.; Chlubná-Eliášová, P.; Greer, H.; Zhou, W.; Seymour, V. R.; Dawson, D. M.; Ashbrook, S. E.; Pinar, A. B.;
McCusker, L. B.; Opanasenko, M.; Čejka, J.; Morris, R. E. Zeolites with Continuously Tuneable Porosity. Angew. Chem., Int. Ed. 2014, 53 (48), 13210-13214.

(22) Evans, J. S. O.; Price, S. J.; Wong, H. V.; O’Hare, D. Kinetic Study of the Intercalation of Cobaltocene by Layered Metal Dichalcogenides with Time-Resolved in Situ X-Ray Powder Diffraction. J. Am. Chem. Soc. 1998, 120 (42), 10837-10846.

(23) Du, Y.; O 'hare, D. Observation of Staging during Intercalation in Layered R-Cobalt Hydroxides: A Synthetic and Kinetic Study. Inorg. Chem. 2008, 47, 11839-11846.

(24) Barroso-Bujans, F.; Alegria, A. Kinetic Differences in the Intercalation of Linear and Cyclic Penta(ethylene Oxide)s into Graphite Oxide Leading to Separation by Topology. Phys. Chem. Chem. Phys. 2017, 19 (28), 18366-18371.

(25) Norby, P. Hydrothermal Conversion of Zeolites: An in Situ Synchrotron X-Ray Powder Diffraction Study. J. Am. Chem. Soc. 1997, 119 (22), 5215-5221.

(26) Rahbani, J.; Khashab, N. M.; Patra, D.; Al-Ghoul, M. Kinetics and Mechanism of Ionic Intercalation/de-Intercalation during the Formation of $\alpha$-Cobalt Hydroxide and Its Polymorphic Transition to $\beta$-Cobalt Hydroxide: Reaction-diffusion Framework. J. Mater. Chem. 2012, 22 (32), 16361.

(27) Saliba, D.; Al-Ghoul, M. Kinetics of Intercalation of Fluorescent Probes in Magnesium-Aluminium Layered Double Hydroxide within a Multiscale Reaction-Diffusion Framework. Philos. Trans. R. Soc., A 2016, 374 (2080), 20160138.

(28) Ragavan, A.; Khan, A.; O’Hare, D. Selective Intercalation of Chlorophenoxyacetates into the Layered Double Hydroxide [LiAl2(OH)6]Cl.xH2O. J. Mater. Chem. 2006, 16 (42), 4155-4159.

(29) Duan, X.; Evans, D. G.; David, G. Layered Double Hydroxides; Springer, 2005.

(30) Millini, R.; Bellussi, G.; Smeets, S.; Xiaodong, Z.; Strohmaier, K. Zeolites in Catalysis: Properties and Applications; Cejka, J., Morris, R. E., Nachtigall, P., Eds.; Royal Society of Chemistry: Croydon, 2017.

(31) Henkelis, S. E.; Mazur, M.; Rice, C. M.; Bignami, G. P. M.; Wheatley, P. S.; Ashbrook, S. E.; Cejka, J.; Morris, R. E. A Procedure for Identifying Possible Products in the Assembly-disassemblyorganization-reassembly (ADOR) Synthesis of Zeolites. Nat. Protoc. 2019, DOI: $10.1038 /$ s41596-018-0114-6. 